\title{
Prognostic factors of postrecurrence survival in completely resected stage I non-small cell lung cancer with distant metastasis
}

\author{
Jung-Jyh Hung, ${ }^{1,2}$ Wen-Juei Jeng, ${ }^{3}$ Wen-Hu Hsu, ${ }^{4}$ Kou-Juey Wu, ${ }^{5}$ \\ Teh-Ying Chou, ${ }^{1}$ Chih-Cheng Hsieh, ${ }^{4}$ Min-Hsiung Huang, ${ }^{4}$ Jung-Sen Liu, ${ }^{2}$ \\ Yu-Chung $\mathrm{Wu}^{4,6}$
}

\begin{abstract}
- A supplementary figure and table are published online only at http://thx.bmj.com/content/ vol65/issue3.

${ }^{1}$ Institute of Clinical Medicine, National Yang-Ming University, Taipei, Taiwan

${ }^{2}$ Department of Surgery, Cathay General Hospital, Taipei, Taiwan ${ }^{3}$ Department of Internal

Medicine, Chang Gung Memorial Hospital, Chang Gung University, Taipei, Taiwan

${ }^{4}$ Division of Thoracic Surgery, Department of Surgery, Taipei Veterans General Hospital, Taipei, Taiwan

${ }^{5}$ Institute of Biochemistry and Molecular Biology, National Yang-Ming University, Taipei, Taiwan

${ }^{6}$ School of Medicine, National Yang-Ming University, Taipei, Taiwan
\end{abstract}

\section{Correspondence to}

Dr Yu-Chung Wu, Division of Thoracic Surgery, Department of Surgery, Taipei Veterans General Hospital, No. 201, Section 2 Shih-Pai Road, Taipei 112, Taiwan; wuyc@vghtpe.gov.tw

J-JH and Y-CW contributed equally to this article.

Received 11 November 2008 Accepted 24 November 2009

\section{ABSTRACT}

Objective Distant metastasis after surgical resection is the most frequent cause of death in patients with nonsmall cell lung cancer (NSCLC). This study aimed to investigate the patterns of distant metastasis and the prognostic factors of postrecurrence survival in patients with resected stage I NSCLC with distant metastases.

Methods The clinicopathological characteristics of 166 patients with distant metastases after complete resection of stage I NSCLC at Taipei Veterans General Hospital between 1980 and 2000 were retrospectively reviewed. The patients were divided into two groups according to patterns of distant metastasis (single or multiple organ metastases). Predictors of postrecurrence survival were analysed.

Results The patterns of distant metastasis included single organ metastasis in 106 (63.9\%) and multiple organ metastases in $60(36.1 \%)$ patients. The 1- and 2-year postrecurrence survival rates for those with single organ metastasis were $30.2 \%$ and $15.1 \%$, respectively. The most common site of single organ metastasis was bone $(32.1 \%)$, followed by the brain $(29.2 \%)$.

Multivariate analysis revealed that disease-free interval $>16$ months (HR 0.534; $95 \%$ Cl 0.288 to 0.990;

$\mathrm{p}=0.046$ ) and treatment for distant metastasis (including re-operation, chemotherapy and/or radiotherapy) (HR $0.245 ; 95 \% \mathrm{Cl} 0.089$ to $0.673 ; p=0.006$ ) were significant predictors of better postrecurrence survival in resected stage I NSCLC with single organ metastasis.

Conclusions A longer disease-free interval is a favourable prognostic predictor for postrecurrence survival in resected stage I NSCLC with single organ metastasis. Treatment for distant metastasis significantly prolongs postrecurrence survival.

\section{INTRODUCTION}

Lung cancer is the main cause of cancer-related death worldwide. For patients with early-stage non-small cell lung cancer (NSCLC), surgical resection is the treatment of choice for curative intent. ${ }^{1-5}$ Tumour recurrence (including local recurrence and distant metastasis) is the most common cause of death, and thus the main obstacle for long-term survival after resection. ${ }^{3-9}$ The postoperative recurrence rates in stage I NSCLC range between $22 \%$ and $38 \%{ }^{3-7}$ In stage I NSCLC after surgical resection, distant metastasis has been reported to be between $14 \%$ and $23 \%{ }^{3-7}$

Even with aggressive multimodality treatments, most patients with distant metastases have little possibility of cure. Several factors reportedly affect survival after distant metastasis, including diseasefree interval, the number of foci of metastatic disease, the organ site of distant metastases, the multiplicity of metastatic organ sites and effects of treatment after distant metastases. ${ }^{11}{ }^{11}$ The majority of patients with resected NSCLC receive chemotherapy, radiotherapy or a combination of therapeutic modalities after distant metastases. ${ }^{6} 91213$ Several studies have been published demonstrating predictors of postrecurrence survival in patients with resected NSCLC after recurrence. ${ }^{3-9}$ However, most of them evaluated postrecurrence survival of patients with a mixture of local or regional recurrence and distant metastases. Few studies focused on postrecurrence survival in patients with distant metastases.

The predictors of postrecurrence survival in resected stage I NSCLC with distant metastasis has not been well demonstrated in the literature. In our previous study, we have demonstrated that patterns of recurrence in resected stage I NSCLC include distant only in $17.8 \%$, local only in $7.9 \%$, and both local and distant in $5.3 \%$ of patients. ${ }^{14}$ Treatment for initial recurrence is a prognostic predictor for postrecurrence survival in resected stage I NSCLC with local recurrence. ${ }^{14}$ In this report, we focused on the patterns of distant metastasis and analysed the prognostic predictors of postrecurrence survival in resected stage I NSCLC with distant metastasis.

\section{PATIENTS AND METHODS}

From January 1980 to December 2000, 970 patients underwent resections for pathological stage I NSCLC at Taipei Veterans General Hospital. The preoperative staging investigation was routinely performed as previously described, ${ }^{14-16}$ including chest and upper abdomen CT scans and bronchoscopy. Nuclear medicine survey of the bone and CT scan of brain were utilised to exclude possible metastases. Mediastinoscopy was performed only when enlarged mediastinal lymph nodes (diameter $>1.0 \mathrm{~cm}$ ) were shown by CT scan. During the study period, positron emission tomography was not a common examination used for staging. Patients with suspected distant metastases were excluded from consideration of thoracotomy. Complete resection of lung cancer and mediastinal lymph nodes dissection/sampling were performed as previously described. ${ }^{14-16}$ No patients had received 
adjuvant therapy after initial surgical resection. Histological typing was based on the WHO classification, ${ }^{17}$ and tumour staging was determined according to the TNM classification of the International System for Staging Lung Cancer. ${ }^{18}$

All patients were followed-up at our outpatient department every 3 months in the first 2 years after resection and at 6 month intervals thereafter. CT scans of chest and upper abdomen were routinely done in every scheduled outpatient department visit for follow-up. Nuclear medicine survey of the bone was arranged every 6 months in the first 2 years after resection and annually thereafter during follow-up. Suspicious bony lesions were confirmed by x-ray or bone biopsy. CT scan of brain was done when neurological symptoms occurred or when clinical suspicions were raised. Once a metastasis was discovered, a routine investigation was arranged to look for other metastatic sites. After initial diagnosis of single or multiple organ distant metastases, further examinations were arranged to discover other metastatic sites if symptoms occurred or clinical suspicions were raised. The hospital charts of all patients were reviewed to collect data of patterns of recurrence, sites of distant metastases and treatment after distant metastasis. Data collected from telephone call and correspondence letters during follow-up were also included.

To investigate their impact on postrecurrence survival, the following clinicopathological factors were used in univariate and multivariate analyses: age at recurrence, gender (male vs female), smoking index, tumour location (right vs left), histological type of the tumour (squamous cell carcinoma vs non-squamous cell carcinoma), tumour size, extent of pulmonary resection (bilobectomy or pneumonectomy vs sublobar resection or lobectomy), T status (T2 vs T1), visceral pleural invasion (present vs absent), disease-free interval ( $>16$ vs $\leq 16$ months), lung metastasis (present vs absent), brain metastasis (present vs absent), bone metastasis (present vs absent), liver metastasis (present vs absent) and treatment for distant metastasis (yes vs no). The number of mediastinal lymph nodes dissected/sampled, including $\mathrm{N} 1$ and N2 nodes, was recorded from pathological reports. Distant metastasis was defined as tumour recurrence in the contralateral lung or outside the hemithorax and mediastinum after surgical resection. Distant only metastasis was defined as only distant metastasis discovered from the initial operation to death or last follow-up. To differentiate second primary tumours from recurrent NSCLC, the criteria proposed by Martini and Melamed were applied. ${ }^{3} 19$ Single and multiple organ metastases were defined as only one or more than one organ site of metastases discovered from initial operation to death or last follow-up, respectively. The length of postrecurrence survival was defined as the interval in months from the date of initial distant metastasis identified to death or the date of the last follow-up.

The postrecurrence survival was calculated by the Kaplan-Meier method. ${ }^{20}$ The $\chi^{2}$ test and the independent sample $t$ test were used to compare groups with respect to categorical and continuous variables as appropriate. For smoking index and disease-free interval, medians and their distributions were compared using Mann-Whitney test. Univariate and multivariate analyses were performed by means of the Cox proportional hazards model using SPSS software (version 12.0; SPSS). The HR of death and its $95 \%$ CI were also calculated. Only variables with a $\mathrm{p}$ value $<0.1$ after the univariate analysis were entered into the multivariate analysis. Statistical significance was defined as $\mathrm{p}<0.05$

\section{RESULTS}

Of the 970 patients, complete follow-up was available for 933 (96.2\%). Among these 933 patients, 166 (17.8\%) developed distant only metastasis after surgical resection. The median follow-up time for these 166 patients was 23.4 months (range 2.1-100.8 months). The median time to initial distant metastasis was 12.1 months (range 0.9-65.1 months). The 1- and 2-year postrecurrence survival rates were $37.7 \%$ and $18.9 \%$, respectively. On the last follow-up session, only three patients were alive, 159 patients died of cancer, one patient died of another cause, and three patients died of unknown causes.

The characteristics of the 166 patients according to patterns of distant metastasis (single or multiple organ metastases) are listed in table 1 . The patterns of distant metastasis included single organ metastasis in 106 (63.9\%) and multiple organ metastases in $60(36.1 \%)$ patients. The single organ metastasis group consisted of more left lung tumour, more squamous cell carcinoma and a lower frequency of visceral pleural invasion. Organ sites of distant metastases and treatment for distant metastasis are listed in table 2 . As compared with the single organ metastasis group, patients with multiple organ metastases were significantly less likely to have liver involvement as the first organ site of distant metastasis $(p=0.013)$. Compared with the multiple organ metastases group, there were significantly more patients without treatment after distant metastasis in the single organ metastasis group $(\mathrm{p}=0.004)$.

The median follow-up time for the 106 patients with single organ metastasis was 21.6 months (range 2.7-100.8 months), while the median time to distant metastasis for this group of patients was 12.6 months (range 1.4-65.1 months). The 1 - and

Table 1 Characteristics of 166 patients with resected stage I non-small cell lung cancer with distant metastasis

\begin{tabular}{|c|c|c|c|}
\hline Variables & $\begin{array}{l}\text { Single organ } \\
\text { metastasis } \\
(n=106)\end{array}$ & $\begin{array}{l}\text { Multiple organ } \\
\text { metastases } \\
(\mathrm{n}=60)\end{array}$ & p Value \\
\hline $\begin{array}{l}\text { Age at initial operation, } \\
\text { years (mean } \pm S D \text { ) }\end{array}$ & $65.8 \pm 8.9$ & $64.4 \pm 9.0$ & 0.328 \\
\hline $\begin{array}{l}\text { Age at recurrence, } \\
\text { years (mean } \pm S D \text { ) }\end{array}$ & $67.2 \pm 9.2$ & $65.4 \pm 9.1$ & 0.239 \\
\hline Sex, n (\%) & & & 0.051 \\
\hline Male & $88(83.0)$ & $42(70.0)$ & \\
\hline Female & $18(17.0)$ & $18(30.0)$ & \\
\hline $\begin{array}{l}\text { Smoking index, pack-years } \\
\text { (median (IOR)) }\end{array}$ & $25.0(0.0-40.0)$ & $14.3(0.0-35.0)$ & 0.180 \\
\hline Tumour location, n (\%) & & & 0.003 \\
\hline Left lung & $39(36.8)$ & $9(15.0)$ & \\
\hline Right lung & $67(63.2)$ & $51(85.0)$ & \\
\hline Tumour size, cm (mean \pm SD) & $4.4 \pm 2.8$ & $4.3 \pm 1.5$ & 0.772 \\
\hline Histological type, n (\%) & & & 0.005 \\
\hline Squamous cell carcinoma & $36(33.9)$ & $10(16.7)$ & \\
\hline Adenocarcinoma & $58(54.7)$ & $37(61.6)$ & \\
\hline Bronchioloalveolar carcinoma & $6(5.7)$ & $1(1.7)$ & \\
\hline Large cell carcinoma & $6(5.7)$ & $9(15.0)$ & \\
\hline Adenosquamous carcinoma & $0(0.0)$ & $3(5.0)$ & \\
\hline Extent of pulmonary resection, $n(\%)$ & & & 0.158 \\
\hline Sublobar resection & $1(0.9)$ & $1(1.7)$ & \\
\hline Lobectomy & $87(82.1)$ & $45(75.0)$ & \\
\hline Bilobectomy or pneumonectomy & $18(17.0)$ & $14(23.3)$ & \\
\hline T status, $\mathrm{n}(\%)$ & & & 0.095 \\
\hline T1 & $34(32.1)$ & $12(20.0)$ & \\
\hline $\mathrm{T} 2$ & $72(67.9)$ & $48(80.0)$ & \\
\hline Visceral pleural invasion, $\mathrm{n}(\%)$ & & & 0.016 \\
\hline Absent & $88(83.0)$ & $39(65.0)$ & \\
\hline Present & $16(15.1)$ & $18(30.0)$ & \\
\hline Unknown & $2(1.9)$ & $3(5.0)$ & \\
\hline $\begin{array}{l}\text { Disease-free interval, } \\
\text { months (median (IQR)) }\end{array}$ & $12.6(6.1-21.9)$ & $10.6(5.2-16.4)$ & 0.118 \\
\hline
\end{tabular}


Table 2 Organ sites of distant metastases and treatment for distant metastasis of 166 patients with resected stage I non-small cell lung cancer with distant metastasis

\begin{tabular}{|c|c|c|c|}
\hline Variables & $\begin{array}{l}\text { Single organ } \\
\text { metastasis } \\
(\mathrm{n}=106)\end{array}$ & $\begin{array}{l}\text { Multiple organ } \\
\text { metastases } \\
(\mathrm{n}=60)\end{array}$ & p Value \\
\hline $\begin{array}{l}\text { First organ site of distant } \\
\text { metastasis, } \mathrm{n}(\%)\end{array}$ & & & 0.019 \\
\hline Lung & $22(20.8)$ & $13(21.7)$ & \\
\hline Brain & $31(29.2)$ & $13(21.7)$ & \\
\hline Bone & $34(32.1)$ & $23(38.2)$ & \\
\hline Liver & $14(13.2)$ & $1(1.7)$ & \\
\hline Adrenal gland & $1(0.9)$ & $4(6.7)$ & \\
\hline Others & $4(3.8)$ & $6(10.0)$ & \\
\hline $\begin{array}{l}\text { Second organ site of distant } \\
\text { metastasis, } n(\%)\end{array}$ & & & NS \\
\hline Lung & - & $15(25.0)$ & \\
\hline Brain & - & $15(25.0)$ & \\
\hline Bone & - & $15(25.0)$ & \\
\hline Liver & - & $8(13.3)$ & \\
\hline Adrenal gland & - & $2(3.3)$ & \\
\hline Others & - & $5(8.4)$ & \\
\hline $\begin{array}{l}\text { Treatment for distant } \\
\text { metastasis, } \mathrm{n}(\%)\end{array}$ & & & 0.009 \\
\hline None & $13(12.3)$ & $1(1.7)$ & \\
\hline With surgery & $9(8.4)$ & $5(8.3)$ & \\
\hline Surgery only & 6 & 2 & \\
\hline Surgery $+R / T$ & 2 & 1 & \\
\hline Surgery $+\mathrm{C} / \mathrm{T}$ and $\mathrm{R} / \mathrm{T}$ & 1 & 2 & \\
\hline $\mathrm{C} / \mathrm{T}$ and/or $\mathrm{R} / \mathrm{T}$ & $39(36.8)$ & $40(66.7)$ & \\
\hline $\mathrm{C} / \mathrm{T}$ only & 13 & 19 & \\
\hline $\mathrm{R} / \mathrm{T}$ only & 21 & 10 & \\
\hline $\mathrm{C} / \mathrm{T}$ and $\mathrm{R} / \mathrm{T}$ & 5 & 11 & \\
\hline Unknown & $45(42.5)$ & $14(23.3)$ & \\
\hline
\end{tabular}

$\mathrm{C} / \mathrm{T}$, chemotherapy; NS, non-significant; $\mathrm{R} / \mathrm{T}$, radiotherapy.

2-year postrecurrence survival rates were $30.2 \%$ and $15.1 \%$, respectively (figure 1). Of these 106 patients, 22 patients $(20.8 \%)$ presented with lung, 31 patients $(29.2 \%)$ presented with brain, 34 patients $(32.1 \%)$ presented with bone, 14 patients $(13.2 \%)$ presented with liver, 1 patient $(0.9 \%)$ presented with adrenal gland and 4 patients (3.8\%) presented with other organ metastases. Treatments for single organ metastasis included surgery for 9 patients, chemotherapy for 13, radiotherapy for 21 and

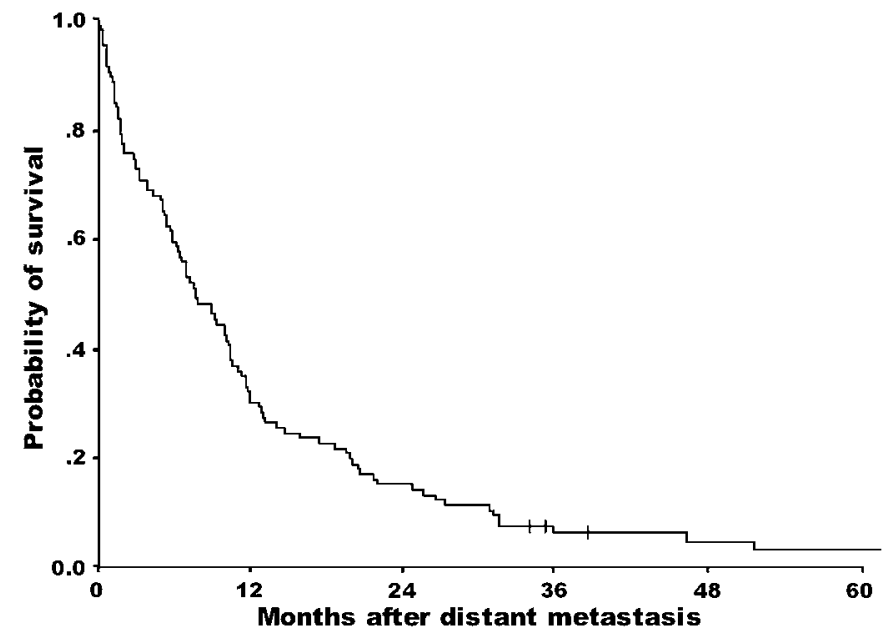

Figure 1 Cumulative probability of postrecurrence survival in 106 patients with resected stage I non-small cell lung cancer with single organ metastasis. a combination of chemotherapy and radiotherapy for 5 patients. The operations for distant metastasis were pulmonary resection in five and craniotomy in another four patients. Two postoperative deaths occurred; both were due to respiratory failure after pulmonary resection. Thirteen patients had no treatment after distant metastasis (eight patients because of poor performance status, one sought alternative treatment, two refused treatment and another two for unknown reasons). Postrecurrence survival in patients with single organ metastasis was not different from that in patients with multiple organ metastases (HR 0.787; 95\% CI 0.569 to $1.089 ; \mathrm{p}=0.133$ ).

Univariate analysis indicated that gender (HR of males 1.905; 95\% CI 1.112 to $3.275 ; \mathrm{p}=0.019$ ), smoking index (HR 1.008; 95\% CI 1.000 to $1.016 ; \mathrm{p}=0.040$ ), histological type (HR of squamous cell carcinoma $1.802 ; 95 \%$ CI 1.183 to $2.740 ; \mathrm{p}=0.006$ ), diseasefree interval (HR of disease-free interval $>16$ months $0.462 ; 95 \%$ CI 0.295 to $0.721 ; \mathrm{p}=0.001$ ), lung metastasis (HR 0.596; $95 \% \mathrm{CI}$ 0.367 to $0.968 ; \mathrm{p}=0.037$ ), bone metastasis ( $\mathrm{HR} 1.553$; $95 \% \mathrm{CI}$ 1.016 to $2.375 ; \mathrm{p}=0.042$ ) and treatment for distant metastasis (HR 0.253; 95\% CI 0.129 to 0.494 ; p $<0.001$ ) had a statistically significant influence on postrecurrence survival in 106 patients with resected stage I NSCLC with single organ metastasis (table 3) (see supplementary table 1 online for complete results of the univariate analysis). Survival was better in women. The hazard of death was greater in patients with a higher smoking index, shorter disease-free interval and squamous cell carcinoma. Patients with lung metastasis had a better postrecurrence survival, while those with bone metastasis had a worse prognosis.

All of the variables listed in table 3 were entered into multivariate analysis. Brain metastasis and liver metastasis were also entered for mutual adjustment despite $p>0.1$. Disease-free interval and treatment for distant metastasis were still

Table 3 Univariate analysis of postrecurrence survival in 106 patients with resected stage I non-small cell lung cancer with single organ metastasis

\begin{tabular}{|c|c|c|c|}
\hline Variables & HR & $95 \% \mathrm{CI}$ & p Value \\
\hline \multicolumn{4}{|l|}{ Gender } \\
\hline Female & 1 & & \\
\hline Male & 1.905 & 1.112 to 3.257 & 0.019 \\
\hline Smoking index, pack-years* & 1.008 & 1.000 to 1.016 & 0.040 \\
\hline \multicolumn{4}{|l|}{ Histological type } \\
\hline Non-squamous cell carcinoma & 1 & & \\
\hline Squamous cell carcinoma & 1.802 & 1.183 to 2.740 & 0.006 \\
\hline \multicolumn{4}{|l|}{ Disease-free interval, months } \\
\hline$\leq 16$ & 1 & & \\
\hline$>16$ & 0.462 & 0.295 to 0.721 & 0.001 \\
\hline \multicolumn{4}{|l|}{ Lung metastasis } \\
\hline Absent & 1 & & \\
\hline Present & 0.596 & 0.367 to 0.968 & 0.037 \\
\hline \multicolumn{4}{|l|}{ Brain metastasis } \\
\hline Absent & 1 & & \\
\hline Present & 0.828 & 0.536 to 1.279 & 0.394 \\
\hline \multicolumn{4}{|l|}{ Bone metastasis } \\
\hline Absent & 1 & & \\
\hline Present & 1.553 & 1.016 to 2.375 & 0.042 \\
\hline \multicolumn{4}{|l|}{ Liver metastasis } \\
\hline Absent & 1 & & \\
\hline Present & 1.321 & 0.733 to 2.380 & 0.354 \\
\hline \multicolumn{4}{|l|}{ Treatment for distant metastasis } \\
\hline No & 1 & & \\
\hline Yes & 0.253 & 0.129 to 0.494 & $<0.001$ \\
\hline
\end{tabular}


significant prognostic indicators in multivariate analysis (table 4). Patients with resected stage I NSCLC with a diseasefree interval $>16$ months (HR 0.534 ; $95 \%$ CI 0.288 to 0.990 ; $\mathrm{p}=0.046$ ) had better survival after distant metastasis than those with a disease-free interval $\leq 16$ months. Patients who had treatment for distant metastasis (HR 0.245; 95\% CI 0.089 to $0.673 ; p=0.006$ ) survived longer than those without treatment. Postrecurrence survival in patients undergoing re-operation was not different from that in patients receiving chemotherapy and/ or radiotherapy $(p=0.085$, log-rank test) (see supplementary figure 1 online).

\section{DISCUSSION}

This study has investigated the patterns of distant metastasis and the prognostic value of conventional clinicopathological factors of postrecurrence survival in resected stage I NSCLC with distant metastases. The patterns of distant metastasis included single and multiple organ metastases in $~ 64 \%$ and $36 \%$ of patients, respectively. Disease-free interval and treatment for distant metastasis were statistically significant predictors of postrecurrence survival in multivariate analysis.

Harpole and co-workers ${ }^{4}$ reported that the initial location of recurrence of stage I NSCLC after surgical resection was at a distant site in $19 \%$, within the ipsilateral hemithorax in $11 \%$ or at both locations in $6 \%$ of patients. In the study of Martini and colleagues, ${ }^{3}$ the incidence of systemic recurrence in patients with resected stage I NSCLC was $20 \%$. Distant recurrence rates between $14 \%$ and $23 \%$ in stage I NSCLC after surgical resection have also been reported in the literature. ${ }^{5-7}$ Our previous study demonstrated that the overall incidence of recurrence in patients with resected stage I NSCLC was $31.0 \%$ (distant only in $17.8 \%$, local only in $7.9 \%$, and local and distant in $5.3 \%) .{ }^{14}$ The current study showed that in $\sim 84 \%$ of patients with distant metastases, these occurred within the first 2 years after operation. A major proportion of patients (62\%) died within 1 year of distant metastasis. Yoshino and co-workers ${ }^{13}$ reported that the 2-year survival rate of patients with NSCLC with postoperative recurrence at distant organs was $15.7 \%$. In the current study, the 2 -year postrecurrence survival rate for patients with resected stage I NSCLC with single organ metastasis was 15.1\%.

Walsh and co-workers ${ }^{21}$ reported that a disease-free interval $>12$ months was a favourable predictor of overall survival and postrecurrence survival in NSCLC after complete resection. Disease-free interval as a significant prognostic factor of postrecurrence survival in NSCLC after complete pulmonary resection has also been reported recently. ${ }^{9} 12$ Our study showed that the disease-free interval was a statistically significant predictor for postrecurrence survival in patients with stage I NSCLC after distant metastasis. A disease-free interval $\leq 16$ months had significant negative effects on postrecurrence survival. Our previous study demonstrated that the number of mediastinal lymph nodes dissected/sampled may be used as an alternative to

Table 4 Multivariate analysis of postrecurrence survival in 106 patients with resected stage I non-small cell lung cancer with single organ metastasis

\begin{tabular}{lcll}
\hline Variables & HR & $\mathbf{9 5 \%} \mathbf{~ C l}$ & p Value \\
\hline $\begin{array}{l}\text { Disease-free interval, months } \\
\leq 16\end{array}$ & 1 & & \\
$>16$ & 0.534 & 0.288 to 0.990 & 0.046 \\
$\begin{array}{l}\text { Treatment for distant } \\
\text { No }\end{array}$ & 1 & & \\
Yes & 0.245 & 0.089 to 0.673 & 0.006 \\
\hline
\end{tabular}

represent the quality of lymphadenectomy in patients with resected stage I NSCLC. ${ }^{15}$ In the current study, the number of mediastinal lymph nodes dissected/sampled was associated with disease-free interval (data not shown). A higher frequency of patients with shorter disease-free interval had fewer mediastinal lymph nodes dissected/sampled. Fewer mediastinal lymph nodes dissected/sampled may lead to less accurate tumour staging and contribute to poor postrecurrence survival through earlier distant metastasis.

Most distant metastases appear as multiple foci in multiple organs after treatment of the original cancer. ${ }^{10}$ Martini and co-workers ${ }^{3}$ reported that the most common site of distant metastasis in patients with resected stage I NSCLC was the brain. Yoshino and co-workers ${ }^{13}$ demonstrated that pulmonary metastasis was most common in patients with NSCLC with recurrence at distant organs, followed by bone metastasis. Our study revealed that bone $(32.1 \%)$ was the most common site of single organ metastasis in patients with resected stage I NSCLC, followed by the brain (29.2\%). We further demonstrated that the patterns of distant metastasis included single and multiple organ metastases in $\sim 64 \%$ and $36 \%$ of patients, respectively. Postrecurrence survival was not significantly different between the two groups of patients. Multiplicity of metastatic organ sites is not a significant prognostic factor in these patients.

Although some reports in the literature had tried to determine the impact of specific distant metastatic organ sites on postrecurrence survival in resected NSCLC, small cohorts or mixtures of local and distant metastasis made it difficult to achieve definite results. Sugimura and colleagues ${ }^{9}$ reported that initial recurrence confined to the lung was associated with better postrecurrence survival in resected NSCLC. Yoshino and co-workers ${ }^{13}$ demonstrated that intrapulmonary metastasis was a favourable factor for postrecurrence survival of resected NSCLC, while bone metastasis was a marginally unfavourable factor. Liver metastasis has also been reported as a worse prognostic factor in NSCLC after recurrence. ${ }^{612}$ Our study focused on postrecurrence survival of single organ metastasis in resected stage I NSCLC. Lung metastasis was associated with better postrecurrence survival in univariate analysis. For the patients with single organ metastasis, patterns of distant metastasis could therefore be divided into two categories according to the organ site of distant metastasis: within the contralateral lung or not. Patients with distant metastases confined within the contralateral lung have significantly better postrecurrence survival than those with distant metastases outside the contralateral lung. The seventh edition of the TNM classification of lung cancer was published in 2009. Better outcomes for patients with contralateral lung nodules were found as compared with distant extrathoracic metastases. Therefore, M1 was recommended to be subclassified, with contralateral lung metastasis as M1a and distant extrathoracic metastases as $\mathrm{M} 1 b .^{22-24}$ Our results support the proposals for the revision of the TNM stage groupings in the seventh edition of the TNM classification of malignant tumours. We further showed that for patients with distant metastases outside the contralateral lung, those with bone metastasis had significantly worse postrecurrence survival.

Treatment for recurrent NSCLC significantly prolongs overall survival and postrecurrence survival. ${ }^{9}{ }^{21}$ Yoshino and colleagues reported that patients who underwent metastatectomy for recurrence in distant organs had significantly longer survival, while those receiving chemotherapy had marginally prolonged survival. ${ }^{13}$ Nakagawa and co-workers ${ }^{6}$ reported that treatment for the initial recurrence prolonged survival in stage I NSCLC after recurrence. In our study, treatment for distant metastasis 
(including surgery and chemotherapy and/or radiotherapy) produced a more favourable survival in resected stage I NSCLC after distant metastasis than no treatment. Eight of the 13 patients without treatment after distant metastasis did not receive treatment due to poor performance status. This may act as a contributory factor to poor prognosis for patients without treatment. There was no significant difference in postrecurrence survival between patients undergoing re-operation and those treated with chemotherapy and/or radiotherapy. However, there were two postoperative deaths due to respiratory failure after pulmonary resection. If the two patients were excluded, patients undergoing re-operation had significantly better postrecurrence survival than those receiving chemotherapy and/or radiotherapy $(p=0.021)$. The therapeutic effects of surgery and chemotherapy and/or radiotherapy need larger series for further investigation.

The multivariate analysis in our study demonstrated that only disease-free interval and treatment for distant metastasis influenced postrecurrence survival. Most variables related to tumour biology were not significant predictors for postrecurrence survival. However, the natural history of lung cancer might be primarily affected by the individual patient's tumour biology which is difficult to predict or classify once systemic disease is evident. Prospective multi-institutional studies are mandatory to validate further the prognostic factors of postrecurrence survival in resected stage I NSCLC after distant metastasis. The impacts of the sequence of organ metastasis in patients with multiple organ metastases and number of foci of metastatic disease also need further investigation.

There are some limitations and biases of this study that should be mentioned. As a retrospective single institute study, patient selection bias and time trend bias regarding the treatment for distant metastasis were inevitable. The criteria of Martini and Melamed ${ }^{319}$ used to distinguish a new primary lung cancer from a metastatic NSCLC have never been validated. There is an inherent bias to define a focus of cancer occurring at any other site $>24$ months later. The definition of an ipsilateral recurrence as a local recurrence also generated inherent bias while differentiating a new primary lung cancer from a recurrent NSCLC.

In conclusion, patients with distant metastasis after initial pulmonary resection are heterogeneous. Longer disease-free interval and treatment for distant metastasis are indicators for better postrecurrence survival in resected stage I NSCLC with single organ metastasis. Aggressive treatment in selected patients with a longer disease-free interval may prolong the postrecurrence survival.

Acknowledgements The authors are grateful to Dr Liang-Shun Wang of En Chu Kong Hospital for his contribution to this article. They also thank Mr Jung-Hsing Lin for his assistance regarding data collection.

Competing interests None.
Provenance and peer review Not commissioned; externally peer reviewed.

\section{REFERENCES}

1. Ichinose $\mathbf{Y}$, Yano $\mathrm{T}$, Asoh $\mathrm{H}$, et al. Prognostic factors obtained by a pathologic examination in completely resected non-small-cell lung cancer. An analysis in each pathologic stage. J Thorac Cardiovasc Surg 1995;110:601-5.

2. Nesbitt JC, Putnam JB Jr, Walsh GL, et al. Survival in early-stage non-small cell lung cancer. Ann Thorac Surg 1995:60:466-72.

3. Martini N, Bains MS, Burt ME, et al. Incidence of local recurrence and second primary tumors in resected stage I lung cancer. J Thorac Cardiovasc Surg 1995; 109:120-9.

4. Harpole DH Jr, Herndon JE II, Young WG Jr, et al. Stage I non-small cell lung cancer Cancer 1995; 76:787-96.

5. Jones DR, Daniel TM, Denlinger CE, et al. Stage IB nonsmall cell lung cancers: are they all the same? Ann Thorac Surg 2006;81:1958-62.

6. Nakagawa T, Okumura N, Ohata K, et al. Postrecurrence survival in patients with stage I non-small cell lung cancer. Eur J Cardiothorac Surg 2008;34:499-504.

7. Al-Kattan K, Sepsas E, Fountain SW, et al. Disease recurrence after resection for stage I lung cancer. Eur J Cardiothorac Surg 1997:12:380-4.

8. Martin J, Ginsberg RJ, Venkatraman ES, et al. Long-term results of combined-modality therapy in resectable non-small-cell lung cancer. J Clin Oncol 2002:20:1989-95.

9. Sugimura H, Nichols FC, Yang $P$, et al. Survival after recurrent nonsmall-cell lung cancer after complete pulmonary resection. Ann Thorac Surg 2007:83:409-18.

10. Cady B. Fundamentals of contemporary surgical oncology: biologic principles and the threshold concept govern treatment and outcomes. J Am Coll Surg 2001:192:777-92.

11. Chambers AF, Naumov GN, Varghese HJ, et al. Critical steps in hematogenous metastasis: an overview. Surg Oncol Clin N Am 2001;10:243-55.

12. Williams BA, Sugimura $\mathrm{H}$, Endo $\mathrm{C}$, et al. Predicting postrecurrence survival among completely resected nonsmall-cell lung cancer patients. Ann Thorac Surg 2006;81:1021-7

13. Yoshino I, Yohena T, Kitajima M, et al. Survival of non-small cell lung cancer patients with postoperative recurrence at distant organs. Ann Thorac Cardiovasc Surg 2001:7:204-9.

14. Hung JJ, Hsu WH, Hsieh CC, et al. Post-recurrence survival in completely resected stage I non-small cell lung cancer with local recurrence. Thorax 2009;64:192-6.

15. Wu YC, Lin CF, Hsu WH, et al. Long-term results of pathological stage I non-small cell lung cancer: validation of using the number of totally removed lymph nodes as a staging control. Eur J Cardiothorac Surg 2003;24:994-1001.

16. Hung JJ, Wang CY, Huang $\mathrm{MH}$, et al. Prognostic factors in resected stage I non-small cell lung cancer with a diameter of $3 \mathrm{~cm}$ or less: visceral pleural invasion did not influence overall and disease-free survival. J Thorac Cardiovasc Surg 2007:134:638-43

17. Travis WD, Colby TB, Corrin B, et al. Histological typing of tumors of lung and pleura. In:Sobin LH, ed. World Health Organization international classification of tumours. 3rd edn. New York: Springer-Verlag, 1999:21-47.

18. Mountain CF. Revisions in the international system for staging lung cancer. Chest 1997:111:1710-7.

19. Martini N, Melamed MR. Multiple primary lung cancers. J Thorac Cardiovasc Surg 1975; 70:606-12

20. Kaplan EL, Meier P. Nonparametric estimation for incomplete observations J Am Stat Assoc 1958;53:457-81.

21. Walsh GL, O'Connor M, Willis KM, et al. Is follow-up of lung cancer patients after resection medically indicated and cost-effective? Ann Thorac Surg 1995:60:1563-70.

22. Goldstraw P, Crowley J, Chansky K, et al. The IASLC lung cancer staging project: proposals for the revision of the TNM stage groupings in the forthcoming (seventh) edition of the TNM classification of malignant tumours. J Thorac Oncol 2007:2:706-14.

23. Postmus PE, Brambilla E, Chansky K, et al. The IASLC lung cancer staging project: proposals for revision of the $\mathrm{M}$ descriptors in the forthcoming (seventh) edition of the TNM classification of lung cancer. J Thorac Oncol 2007;2:686-93.

24. Groome PA, Bolejack V, Crowley JJ, et al. The IASLC lung cancer staging project: validation of the proposals for revision of the $T, N$, and $M$ descriptors and consequent stage groupings in the forthcoming (seventh) edition of the TNM classification of malignant tumours. J Thorac Oncol 2007:2:694-705. 